\title{
SPARSITY ASSISTED SIGNAL SMOOTHING IN TIME-SERIES SEISMIC DATA
}

\author{
Abhishek Kesharwani, Devika Chib and Shubham Singh \\ Third Year (B-Tech), Vellore Institute of Technology, Vellore, Tamil Nadu, India \\ Dr. Malaya Kumar Hota \\ Professor, Department of Communication Engineering, School of Electronics Engineering, \\ Vellore Institute of Engineering, Vellore, Tamil Nadu, India
}

\begin{abstract}
Sparsity-assisted signal smoothing (SASS) is dependent on a banded matrix formulation of the recursive filtering of finite length input signals. This paper presents a formulation of higher-order zero-phase lowpass, high-pass, and band-pass infinite impulse response filters as matrices, using the spectral transformation of the statespace representation of digital filters, that avoids the unwanted transient artifacts at signal end-points in the original formulation and denoises marine seismic signals. The simulations have been created by running the code in MATLAB, using synthesized data, thus the results can be compared with the other methods easily. The problems posed while noise removal by conventional methods are also discussed. We have tried our best in presenting this new technology and the output obtained for the same clearly indicates the effectiveness and robustness of a SASS denoising algorithm and has a wide scope of application in the future, which can be a very important asset to the research process.
\end{abstract}

Key words: Sparsity-assisted signal smoothing, banded matrix formulation, low-pass, high-pass, band-pass filter.

Cite this Article: Abhishek Kesharwani, Devika Chib, Shubham Singh and Dr. Malaya Kumar Hota, Sparsity Assisted Signal Smoothing in Time-Series Seismic Data. International Journal of Electrical Engineering and Technology, 11(5), 2020, pp. 64-72.

http://iaeme.com/Home/issue/IJEET?Volume=11\&Issue $=5$

\section{INTRODUCTION}

The presence of Noise in marine seismic signals is a major menace and must be removed for the data to be further processed. This noise is incoherent along the spatial direction. Hence, there are several methods that have been developed to tackle this problem.

Signal denoising of time-series data is a very widely used technology and many areas of science are implementing it extensively in research these days. One of its many popular uses, 
is in the electroencephalogram (EEG) data, which is useful in cognitive neuroscience and diagnosis.

The most trot-on-path to do this is by the use of linear time-invariant (LTI) filters. The conventional way of recognizing patterns in time-series involves the following steps: feature extraction, feature selection, and classification. If the frequency band of the target signal is known then LTI is the best way to go about it. However, if the signal consists of discontinuities, it fails by over smoothing the discontinuous areas. In such cases, fast iterative methods such as total-variation denoising (TVD) [16] are more suitable.

A novel time series signal analysis method in seismic exploration is the Hilbert-Huang transform (HHT), which integrates classical Hilbert transform (HT) and empirical mode decomposition (EMD), which expresses the intrinsic essence using a clear and simple algorithm. But there exists a serious mode mixing phenomenon in EMD. To solve this mode mixing problem of EMD, ensemble empirical mode decomposition (EEMD) which is a noiseassisted data analysis method is adopted. It skillfully solves the mode mixing problem by adding white noise to the original signal. EEMD holds the ability to decompose the signal into different IMFs and analyze the time-frequency distribution of the signal. The time-frequency spectrum that is obtained by EEMD more realistically reflects the actual geology than by EMD.

Although, EEMD greatly reduced the possibility of mode mixing, it raises the issue of a nonnegligible residue noise that is mixed into the original signal after reconstruction, if the number of trails is not large enough.

An alternative is the use of wavelets, it offers an orthogonal multiresolution representation of the signals and has advantages over traditional Fourier methods of signal analysis that contains discontinuities. It uses adaptive thresholding of wavelength coefficients which can simultaneously denoise and preserve the singularity points. The major drawback of the use of wavelets is that it introduces pseudo-Gibbs artifacts at the singular points due to oscillations that are more local and of smaller amplitude near the signal's discontinuities.

One more popularly used technology is by decomposing the underlying signal as the sum of two or more components which include a polynomial signal or low-frequency signal together with a sparse or its derivative signal.

Sparsity-assisted signal smoothing also called SASS [2], was developed for the sole purpose of filtering out a signal that had discontinuities in its derivative (of some designated order) but it is smooth otherwise. SASS combines and unifies the conventional LTI low-pass filtering and, generalized TV denoising. Hence, SASS is very useful for a wider class of signals other than, either LTI low-pass filtering or TV denoising alone. The SASS algorithm formulates the denoising problem as a sparse deconvolution problem, and in turn, as an optimization problem comprising a data fidelity term and a sparse regularization term. The SASS problem formulation is expressed in the terms of banded Toeplitz matrices. Furthermore, the computationally efficient implementations of SASS rely on the fast solvers for banded systems of linear equations.

In this paper, we are introducing an improved version of SASS, specifically for the denoising of seismic data. Previous formulations give rise to unwanted transient artifacts at the start and end of finite-length data [2,3]. Ad-hoc pre-processing of the input data is done to avoid these artifacts which limits the utility of SASS, especially for the filtering out of short input data. The latest matrix formulation that is presented below does not give rise to transient artifacts at all, hence no ad-hoc pre-processing is needed and SASS can be effectively applied to both the short and the long finite-length data. 
We seek to introduce a new approach by implementing the design of zero-phase noncasual filters as matrices, into marine signal denoising.

\subsection{Relation to Prior Work}

Several prior works have studied many of the signal models that are considered here, like, a signal comprising the sum of low-frequency signals and sparse-derivative signals $[4,5,6,7,8,3]$. The most closely related and relevant work to this, is by Gholami and Hosseini [5], who combine Tikhonov (quadratic) regularization and TV denoising. With due contrast, SASS is formulated explicitly in terms of an LTI low-pass filter, which is reduced as a special case, and hence, can be understood in terms of its frequency responses; while, the method is formulated in terms of the famous Tikhonov regularization. In addition to this, SASS is formulated in such a way, to allow a higher-order sparse derivative and exploits a factorization without which the estimated sparse-derivative signal component may tend to be unbounded in the higher-order case, which in return would hinder the usability of the result and impede numerical stability of the optimization algorithms. We note that it also considers the problems of deconvolution and compressed sensing, which are clearly not considered here.

As SASS is considered to be an extension of TV denoising, we can note that several extensions of TV denoising have been proposed all around $[9,10,11,12]$. In contrast to these known methods, SASS can also be considered to be an extension of LTI filtering. SASS unifies the TV denoising and LTI filtering, and hence it conforms to and builds upon elementary signal processing.

Wavelet-based denoising method is considered suitable for the type of piecewise-smooth signal that is used here. But this simple wavelet-domain denoising may lead to artifacts; hence, wavelet-based signal models have been developed to explicitly account for certain singularities (i.e., discontinuities in the derivative of a signal), such as: wavelet footprints[13,3], singularity detection[14,15], TV-wavelet [16,17], hidden Markov tree [18], and singularity approximation [19,20]. Although SASS is used less (generally) than waveletbased methods (it does not have a multiscale property), it is simpler than wavelet-based methods because it doesn't involve a wavelet-domain singularity-based modelling. SASS preserves sparse singularities, in an otherwise smooth signal without inducing wavelet-like artifacts.

\section{SPARSITY ASSISTED SIGNAL SMOOTHING}

We assume the signal $x$ to be estimated can be expressed as

$$
x(n)=x_{1}(n)+x_{2}(n), n \in Z
$$

where signal $\boldsymbol{x}_{1}$, has a very sparse K-order derivative and $x 2$ here, is a low frequency signal. We assumed the signal $x$ is corrupted by the Additive White Gaussian Noise (AWGN),

$$
y(n)=x(n)+w(n), n \in Z
$$

where w, is the noise. We assumed that, if the sparse-derivative component $\boldsymbol{x}_{\boldsymbol{1}}$ was absent, then in that case, low-pass filtering would be sufficient to estimate $\boldsymbol{x}_{2}$ (since $\boldsymbol{x}_{2}$ is a lowfrequency signal, by assumption). Hence, if $\boldsymbol{x}_{1}$ was known, we may have estimated $\boldsymbol{x}_{\boldsymbol{2}}$ by subtracting $\boldsymbol{x}_{\boldsymbol{1}}$ from the noisy data $\boldsymbol{y}$ and low-pass filtering, i.e.,

$$
x_{2}=L P F\left\{y-x_{1}\right\}
$$


where LPF is representing a zero-phase, low-pass filter. So, we have proposed to estimate $\boldsymbol{x}$ as

$$
\widehat{x}=\widehat{x_{1}}+\operatorname{LPF}\{y-\hat{x}\}
$$

where $\widehat{\boldsymbol{x}}_{\boldsymbol{1}}$, is an estimate of $\boldsymbol{x}_{1}$. Since our low-pass filter is linear, we may write

$$
\begin{gathered}
\hat{x}=\hat{x}_{1}+\operatorname{LPF}\{y\}-\operatorname{LPF}\{x\} \\
=\operatorname{LPF}\{y\}+\operatorname{HPF}\left\{\hat{x}_{1}\right\}
\end{gathered}
$$

where HPF is representing the zero-phase high-pass filter I-LPF where I, is identity operator. Using the low-pass filter $\mathrm{H}$ and the corresponding high-pass filter $\mathrm{G}$, we have

$$
\hat{x}=A^{-1} Q^{T} Q y+\alpha A^{-1} P^{T} P_{1} u
$$

where $\boldsymbol{x}_{\boldsymbol{l}}$, is a signal with sparse K-order derivatives, i.e., $D x_{1}$ is sparse. Let's use $P(z)$ and $G(z)$ with parameters such as $d$ with $\mathrm{K} 6 \mathrm{~d}$. Then $P=P_{1} D$ from, hence we can write

$$
\hat{x}=A^{-1} Q^{T} Q y+\alpha A^{-1} P^{T} P_{1} u
$$

Since $D x_{1}$ is sparse, we can write the signal model as

$$
\hat{x}=A^{-1} Q^{T} Q y+\alpha A^{-1} P^{T} P_{1} u
$$

where $\mathrm{u}$ is sparse (and is yet to be determined/optimized).

Hence, a favorable cost function to determine $\boldsymbol{u}$ is

$$
J(u)=\frac{1}{2}\left\|y-A^{-1} Q^{T} Q y-\alpha A^{-1} P^{T} P_{1} u\right\|_{2}^{2}+\lambda\|u\|_{1}
$$

where the ' 1 norm is being used to induce sparsity of $u$. The quadratic data fidelity term used here, corresponds to the additive white Gaussian noise assumption. We have here

$y-A^{-1} Q^{T} Q y=\alpha A^{-1} P^{T} P y$, i.e,

$$
y-L P F\{y\}=H P F\{y\}
$$

so the cost function to be minimized may be written as

$$
J(u)=\frac{\alpha^{2}}{2}\left\|A^{-1} P^{T}\left(P y-P_{1} u\right)\right\|_{2}^{2}+\lambda\|u\|_{1}
$$

To clarify and/or summarize the matrix sizes: If $y \in R^{N}$, then 
$u \in R^{N-K}, P_{1} \in R^{(N-d) X(N-K)}$

$A \in R^{N X N}, P \in R^{(N-d) X N}$

The minimization of cost function $\mathrm{J}$ is the standard '1-norm sparse least squares problem arising in basis pursuit denoising, compressed sensing, etc. It may be solved by the iterative shrinkage/thresholding algorithm (ISTA) (an instance of forward-backward splitting), accelerated variants of ISTA (FISTA, FASTA, etc.), alternating the direction method of multipliers (ADMM), iterative reweighted least-squares (IRLS), etc. Therefore, we can omit details about how to perform the minimization of the cost function $\mathrm{J}$. We only take note that all matrices $A, P$, and $P_{1}$ are banded; thus, algorithms may be implemented with high computational efficiency [2]. A key point in the this above mentioned formulation is that, even though we model $D x_{1}$ as sparse, the matrix $D$ doesn't appear in the '1-norm penalty term of cost function. Instead of penalizing $D x_{1}$, we penalize $u$. This simplifies the problem and its algorithmic solution. This simplification is possible because $D$ is a factor of $P$, i.e., $D(z)$ is a factor of $P(z)$.

\section{EXAMPLE}

As shown in Fig. 1, Fig. 1(a) denotes a sinusoid signal $S_{l}=\sin 20 \pi t$, Fig. 1(b) represents an intermittent signal

$$
S_{2}=\left\{\begin{array}{cc}
0.2 \times \sin (300 \pi t) & \text { when } 0.05 \leq t \leq 0.1 \\
0.4 \times \sin (100 \pi t) & \text { when } 0.15 \leq t \leq 0.25 \\
0 & \text { other }
\end{array}\right.
$$

and Fig. 1(c) shows the signal $S=S_{1+} S_{2}$.
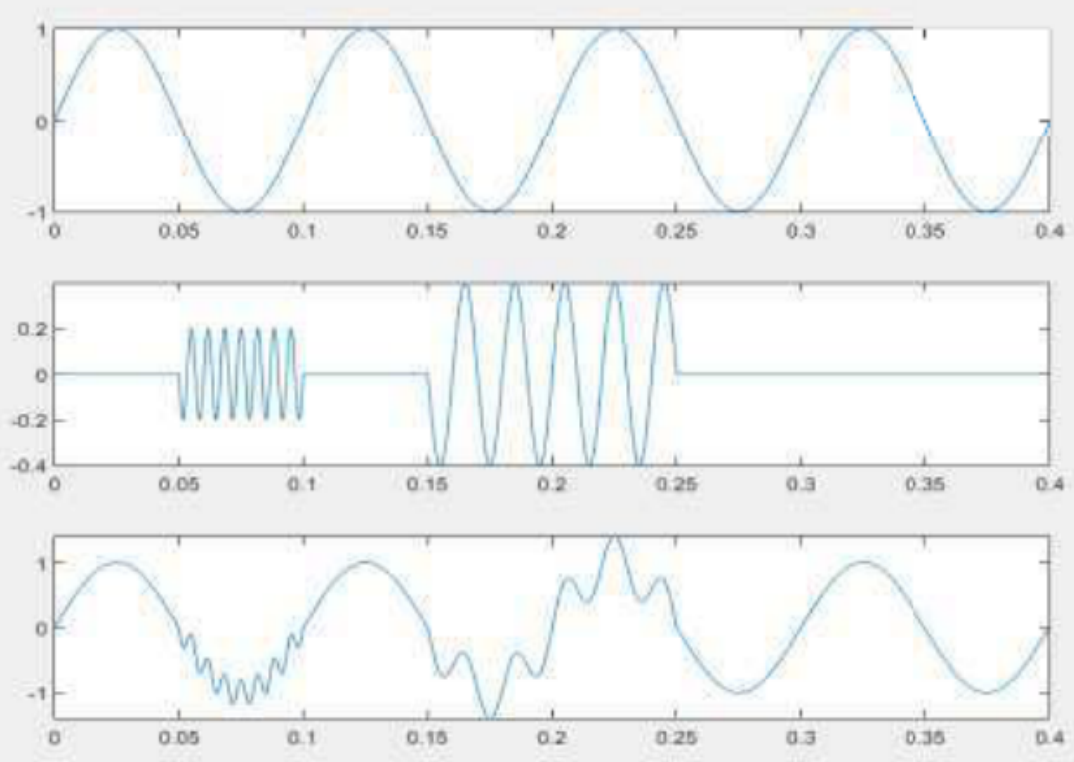

Figure 1 Synthesized signal: (a) signal s1; (b) signal s2; (c) signal s=s1+s2 
To signal S white gaussian noise of standard deviation 0.1 is added, as shown in Fig. 2(a).

The output of SASS, as shown in Fig. 2(b), accurately preserves the sharp peaks of the seismic waveform as we expected. Indeed, the first-order derivatives of the SASS-denoised signals have jump discontinuities in a lot of places. The sparse signal called $\mathrm{u}$, which is computed as a part of the method, is shown in Fig. 2(c). It is these non-zero values of $u$ that are to produce the jump discontinuities of the first-order derivatives of the denoised signals. The denoised signal that we talk about is given, where $\mathrm{u}$ is obtained by minimizing the cost function. We have already set the value of the parameter $\lambda$ by using the method that was suggested.
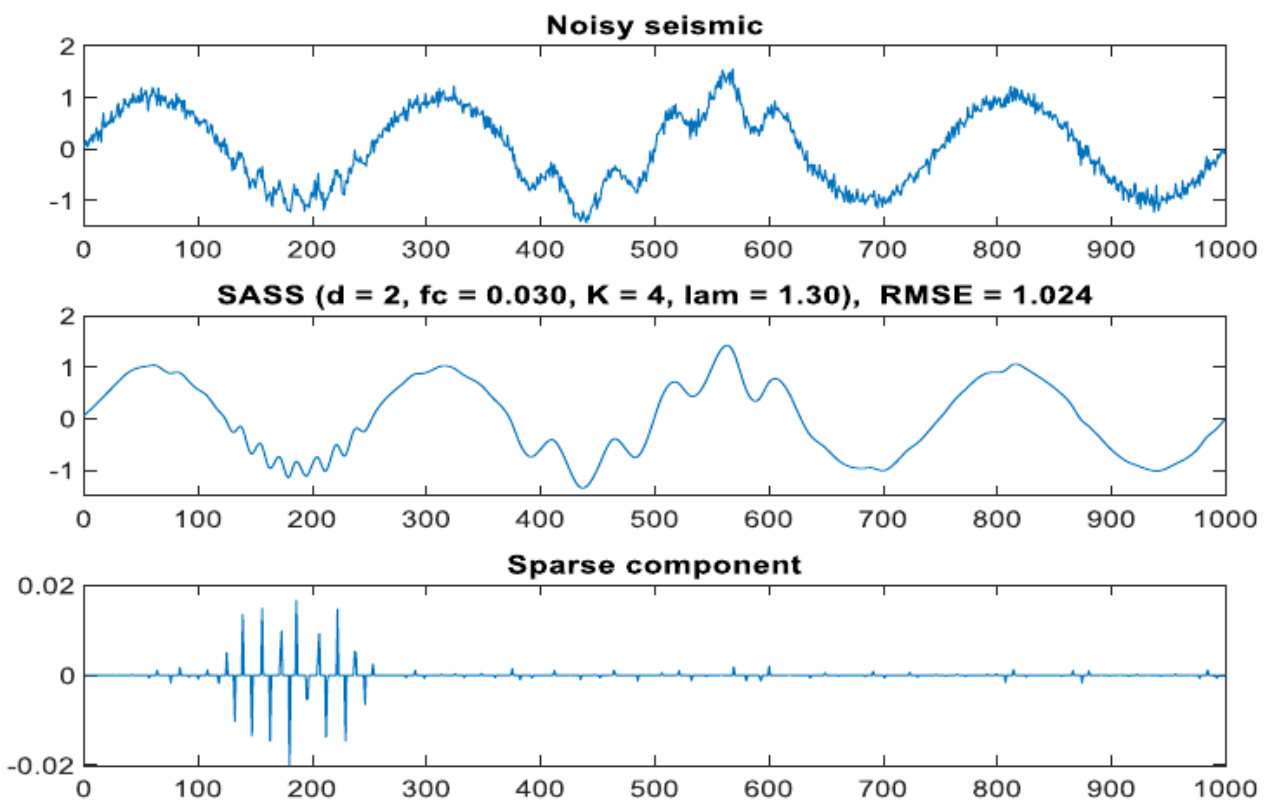

Figure 2 Noisy Signal: (a) signal $\mathrm{S}$ after addition of white noise; (b) output of SASS at d=2, K=4; (c) sparse component

Hence to demonstrate the applications of our novel filter designs, we propose a signal model that denoises and identifies patterns simultaneously. We use our proposed design to denoise the seismic reflection signals. We test an existing signal model that simultaneously combines LTI filters and sparsity-based methods.

Because the zero-phase filters in the SASS signal model are stable, hence they demonstrate consistent results on changing the orders of the filter.

The parameters are, d: degree of the filter; fc: cut-off frequency (normalized frequency); $\mathrm{N}$ : length of the signal; K: order of difference matrix D. As you can see for yourself that the noise is considerably reduced, and can be fine-tuned even more as per the needs of the researcher. This proposed idea can change the conventional ways of doing things. 

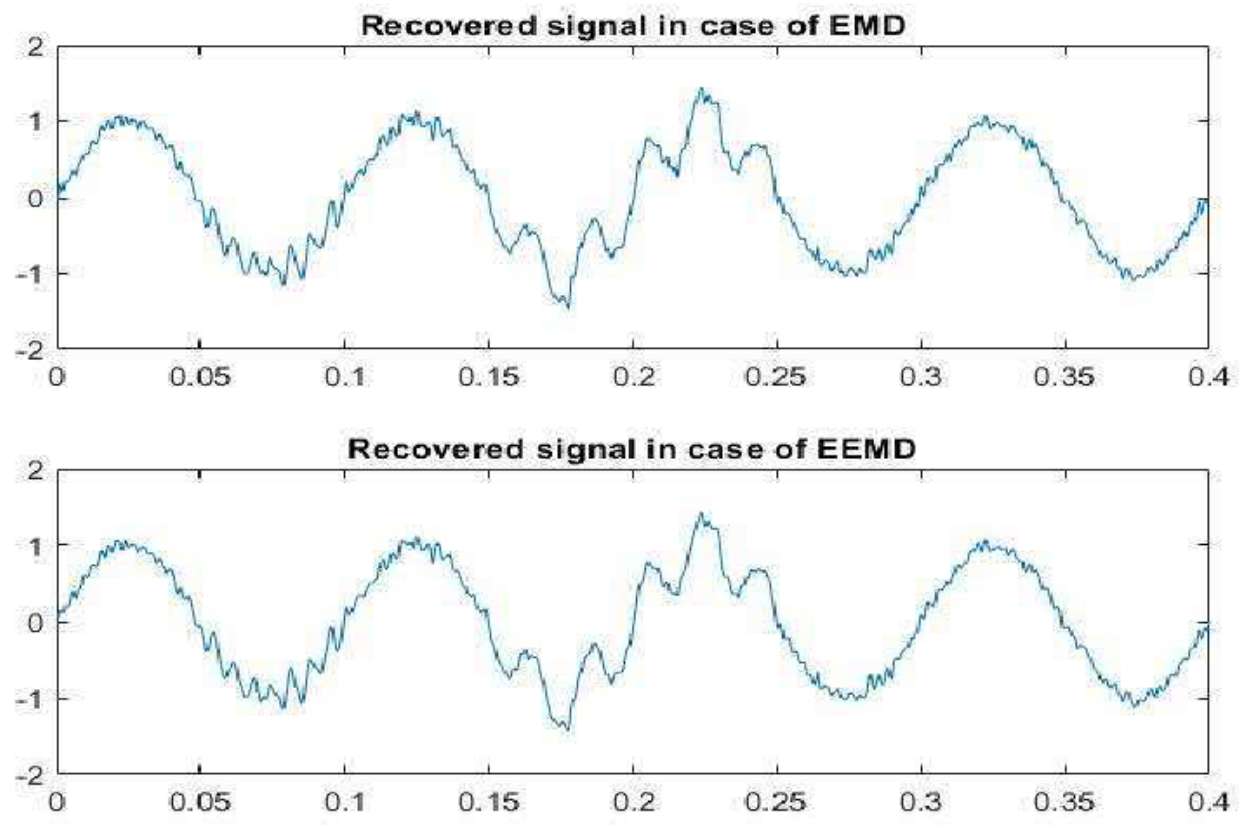

Figure 3 Denoised signal obtained using: (a) EMD (b) EEMD

Comparing figures 2(b) , 3(a) and 3(b), it can clearly be seen that SASS algorithm is more effective in denoising the signal as opposed to EMD and EEMD algorithms.

Further, we compare the SNR values, which is a measure of the relativeness of signal strength to background noise. It may also be defined as the ratio of signal power to noise power.

We use the following formulae to arrive at the SNR values:

$$
\begin{aligned}
& (S N R)_{\text {input }}=\frac{\sum \mid \text { signal }\left.\right|^{2}}{\sum \mid\left.(\text { noisy signal-signal })\right|^{2}} \\
& (S N R)_{\text {output }}=\frac{\sum \mid \text { signal }\left.\right|^{2}}{\sum \mid\left.(\text { signal-recovered signal })\right|^{2}} \\
& (S N R)_{d B}=10 \log (S N R) \\
& (S N R)_{\text {improved in } d B}=(S N R)_{\text {input in } d B}-(S N R)_{\text {output in } d B}
\end{aligned}
$$

The obtained improved SNR values of EMD, EEMD, and SASS, were 11.112, 12.779 and 20.962, respectively.Hence, it can be seen that the SNR ratio obtained using SASS is much higher than that of both EMD and EEMD.

\section{CONCLUSION}

We propose a SASS formulation for denoising marine seismic reflection signals. We managed to combine conventional LTI low-pass, high-pass filtering into a design of zerophase non-casual filters as matrices. This helped us simplify a marine signal denoising problem into a sparse deconvolution problem. The SASS problem formulation is expressed in the terms of banded Toeplitz matrices. Unlike, previous formulations where Ad-hoc preprocessing had to be done to get rid of unwanted transient artifacts at signal end-points of finite length data, the method demonstrated in this paper does not give rise to any such endsignal artifacts and thus, no pre-processing is required. Optimization of the signal was achieved by manipulating a data fidelity term and a sparse regularization term. Proposed method has high computational efficiency due to a banded system of linear equations. 
Furthermore, the signal can be fine-tuned parametrically, as per requirement, hence providing greater control and flexibility over the denoising process. When compared to other marine seismic signal denoising methods like EMD and EEMD, SASS gives a more smoothened output. The SNR value obtained using Sparsity-Assisted Signal Smoothing, is much higher than that of both EMD and EEMD, which ensures minimal transmission loss due to random noises. The data used here was synthesized using MATLAB, however this method can be applied to real-life data as well.

\section{REFERENCES}

[1] W. Selesnick et al., (2015) Excursions in Harmonic Analysis, Birkhäuser Basel, vol. 4, pp. 149-176

[2] W. Sclesnick, S. Arnold and V. R. Dantham, (2012) "Polynomial smoothing of time series with additive step discontinuities", IEEE Trans. Signal Process., vol. 60, no. 12, pp. 63056318, December

[3] D. Van, De Ville, B. Forster-Heinlein, M. Unser and T. Blu, (2010) "Analytical footprints: Compact representation of elementary singularities in wavelet bases", IEEE Trans. Signal Process., vol. 58, no. 12, pp. 6105-6118

[4] Y. Ding and I. W. Selesnick, (2016) Sparsity-based correction of exponential artifacts", Signal Processing, vol. 120, pp. 236-248, March

[5] Gholami and S. M. Hosseini, (2013) "A balanced combination of Tikhonov and total variation regularizations for reconstruction of piecewise-smooth signals", Signal Processing, vol. 93, no. 7 , pp. $1945-1960$

[6] X. Ning, I. W. Selcsnick and L. Duval, (2014) "Chromatogram baseline estimation and denoising using sparsity (BEADS)", Chemometrics and Intelligent Laboratory Systems, vol. 139, pp. 156-167, December

[7] W. Sclesnick, H. L. Graber, Y. Ding, T. Zhang and R. L. Barbour, (2014) "Transient artifact reduction algorithm (TARA) based on sparse optimization", IEEE Trans. Signal Process., vol. 62, no. 24, pp. 6596-6611, December

[8] W. Sclesnick, H. L. Graber, D. S. Pfeil and R. L. Barbour, (2014) "Simultaneous low-pass filtering and total variation denoising", IEEE Trans. Signal Process., vol. 62, no. 5, pp. 11091124, March

[9] K. Bredies, K. Kunisch and T. Pock, (2010) "Total generalized variation", SIAM J. Imag. Sci., vol. 3, no. 3, pp. 492-526

[10] Y. Hu and M. Jacob, (2012) "Higher degree total variation (HDTV) regularization for image recovery", IEEE Trans. Image Process., vol. 21, no. 5, pp. 2559-2571, May

[11] F. I. Karahanoglu, I. Bayram and D. Van De Ville, (2011) "A signal processing approach to generalized 1-d total variation", IEEE Trans. Signal Process., vol. 59, no. 11, pp. 5265-5274, November

[12] S.-H. Lee and M. G. Kang, (2007) "Total variation-based image noise reduction with generalized fidelity function", IEEE Signal Processing Letters, vol. 14, no. 11, pp. 832-835, November

[13] P. L. Dragotti and M. Vetterli, (2003) "Wavelet footprints: theory algorithms and applications", IEEE Trans. Signal Process., vol. 51, no. 5, pp. 1306-1323, May

[14] T.-C. Hsung, D. P. Lun and W.-C. Siu, (1999) "Denoising by singularity detection", IEEE Trans. Signal Process., vol. 47, no. 11, pp. 3139-3144 
Abhishek Kesharwani, Devika Chib, Shubham Singh and Dr. Malaya Kumar Hota

[15] B. Jalil, O. Beya, E. Fauvet and O. Laligant, (2011) "Subsignal-based denoising from piecewise linear or constant signal", Optical Engineering, vol. 50, no. 11, pp. 117004(1-14), November

[16] S. Durand and J. Froment, (2003) "Reconstruction of wavelet coefficients using total variation minimization", SIAM J. Sci. Comput., vol. 24, no. 5, pp. 1754-1767

[17] S. Durand and M. Nikolova, (2007) "Denoising of frame coefficients using $\ell 1$ data-fidelity term and edge-preserving regularization", Multiscale Modeling \& Simulation, vol. 6, no. 2, pp. $547-576$

[18] M. S. Crouse, R. D. Nowak and R. G. Baraniuk, (1998) "Wavelet-based signal processing using hidden Markov models", IEEE Trans. Signal Process., vol. 46, no. 4, pp. 886-902, April

[19] V. Bruni, B. Piccoli and D. Vitulano, (2008) "A fast computation method for time scale signal denoising", Signal Image and Video Processing, vol. 3, no. 1, pp. 63-83

[20] V. Bruni and D. Vitulano, (2006) "Wavelet-based signal de-noising via simple singularities approximation", Signal Processing, vol. 86, no. 4, pp. 859-876, April 